\title{
Sensor data identification based reagent cabinet management system
}

\author{
Changsu Kim, Hongyoul Kim, and Hoekyung Jung
}

Department of Computer Engineering, Pai Chai University, South Korea

\begin{tabular}{l} 
Article Info \\
\hline Article history: \\
Received Apr 21, 2019 \\
Revised Jul 6, 2019 \\
Accepted Jul 17, 20 \\
\hline Keywords: \\
Embedded System \\
IoT \\
Reagent \\
Remote Control \\
Sensor
\end{tabular}

Article Info

Article history:

Received Apr 21, 2019

Revised Jul 6, 2019

Keywords:

Embedded System

Reagent

Sensor

\begin{abstract}
Recently, a reagent cabinet is used in a laboratory or a laboratory is required to have a system capable of identifying a dangerous situation through sensor data as various sensors are utilized. The existing system identifies the dangerous situation through various sensor data, but there is a problem that the server performs all the operations and the operation of the device is performed manually. In order to solve this problem, this paper proposes a system that can identify the dangerous situation and automatically operate the equipment through the internal environment data of the reagent cabinet. Identification of the hazardous situation is done through the master node used in the reagent cabinet, not the server. The server can continuously update the sensor data through the master node and monitor the real-time status of the reagent cabinet through the application. In this way, it is expected that the risk situation will be promptly addressed by identifying the dangerous situation in the reagent cabinet and operating the device.
\end{abstract}

Copyright () 2019 Institute of Advanced Engineering and Science. All rights reserved.

\section{Corresponding Author:}

Hoekyung Jung, Departement of Computer Engineering,

Pai Chai University, 155-40 Baejae-ro, Seogu, DaeJeon, South Korea.

Email: hkjung@pcu.ac.kr

\section{INTRODUCTION}

Recently, reagent cabinet management systems have been studied to prevent or cope with safety accidents occurring in laboratories[1-3]. The reagent cabinet management system measures the internal environment of the reagent cabinet through gas sensors and environmental sensors, or measures toxic gases that may occur inside the reagent cabinet[4-7]. Also, the sensor data is used to check whether a dangerous situation occurs in the reagent cabinet. In addition, the application or embedded board enables real-time monitoring and control of the state of the reagent cabinet[8-10].

The existing reagent cabinet safety management system is used to measure the internal environment of the reagent through environment sensor, gas sensor, and other sensors and transmit it to the server and confirms the occurrence of a dangerous situation. When a dangerous situation is identified, the manager manually controls the device through the transmitted notification message[11-13]. However, since the manager manually manages the dangerous situation, a safety accident can occur because the manager does not take prompt action when the manager is absent. In addition, since the server handles all the work, managing a large number of reagent cabinets may not be able to identify dangerous situations or send notification messages quickly[14-16].

In order to solve this problem, we propose a system that identifies a dangerous situation in a reagent cabinet and automatically selects a device that can cope with it and then sends an operation command automatically. To identify hazardous situations in the reagent cabinet, a sensor node collects the internal environmental data of the reagent cabinet and a master node performs dangerous situation identification and device control command operations through the collected sensor data. The master node identifies a dangerous 
situation through the sensor data transmitted every second from the sensor node. Also, the master node selects the device that can cope with the identified dangerous situation and send the operation command automatically. Therefore, if a dangerous situation occurs, it will be treated promptly and safe reagent management will be achieved.

\section{SYSTEM DESIGN}

This system collects the internal environmental data of the reagent cabinet and can automatically operate the device in case of a dangerous situation. The proposed system uses a sensor node that collects sensor data and a master node that identifies a dangerous situation through collected sensor data and sends operation commands to the device. The sensor node measures the internal environmental data of the reagent cabinet every second and continuously transmits the sensor data to the master node. The master node identifies the dangerous situation through the received sensor data and controls the device. In addition, sensor data is transmitted to the server to update sensor data in real time. Figure 1 shows the structure of the system.

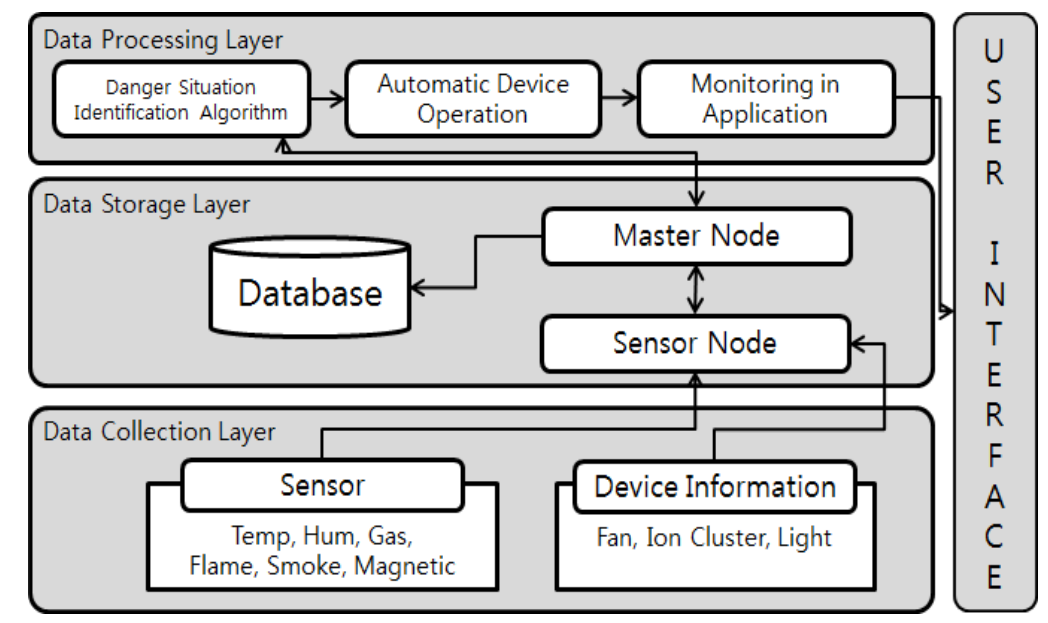

Figure 1. System architecture

The data collection layer collects data about sensors and devices connected with sensor nodes. The sensors utilize temperature, humidity, gas (Hydrogen Sulfide, Nitrogen Dioxide, and Sulfide Dioxide Gas), fire detection, smoke detection, and magnetic sensors. The instrument uses a fan, an ion cluster, and a lamp. Information about sensors and devices is measured and collected every second and then sent to the master node. The database of the data storage layer updates the sensor data by continuously transmitting the collected sensor data to the server through the master node. The data processing layer performs tasks such as identification of the dangerous situation performed by the master node and automatic control of the device. In addition, the operation of the device and the real-time status of the reagent can be remotely monitored through the application.

Figure 2 shows the operation of the device according to temperature. The dangerous situation due to temperature is checked based on the average value of the minimum threshold and the maximum threshold set by the manager.

First, if the temperature of the reagent cabinet is higher than the average value, distinguish whether the current temperature is higher or lower than the maximum threshold. If it is higher than the maximum threshold, identify a dangerous situation and continuously check the fire detection sensor to see if a fire has occurred. In addition, the fan speed is set to maximum $(1.0 \mathrm{~m} / \mathrm{s})$ and a warning message is sent to the manager. On the other hand, if the temperature is lower than the maximum temperature value, set the fan speed to the basic value $(0.53 \mathrm{~m} / \mathrm{s})$.

Second, if the temperature of the reagent cabinet is lower than the average value, check whether the current temperature is lower or higher than the minimum threshold. If it is lower than the minimum threshold value, identify it as a dangerous situation and set the fan speed to minimum $(0.1 \mathrm{~m} / \mathrm{s})$ and send a warning message to the manager. Also, if it is higher than the minimum threshold value, set the fan speed to the basic $(0.53 \mathrm{~m} / \mathrm{s})$. 
Figure 3 shows the operation of the device according to the humidity. Humidity controls the instrument through a total of five situations. First, if the humidity value is above $80 \%$, set the fan speed to maximum $(1.00 \mathrm{~m} / \mathrm{s})$ and send a warning message to the manager to check the condition of the reagent cabinet. If the humidity is more than $40 \%$ and less than $80 \%$, set the fan at the maximum wind speed $(1.00 \mathrm{~m} / \mathrm{s})$ so that the moisture inside the reagent cabinet can escape. If the humidity is not less than $30 \%$ and less than $40 \%$, the fan speed is set to a basic value $(0.53 \mathrm{~m} / \mathrm{s})$ because the threshold value is not exceeded. If the humidity is more than $10 \%$ and less than $30 \%$, the fan speed is set to the minimum $(0.1 \mathrm{~m} / \mathrm{s})$. If the humidity is less than $10 \%$, the operation of the fan stops.

Figure 4 shows the operation of the device according to hydrogen sulfide. If the hydrogen sulfide value is more than $10 \mathrm{ppm}$, the device activates the fan and ion cluster and locks the door so that the reagent cabinet door cannot be opened from the outside. Also, a warning message is sent to the manager to inform the current state of the reagent cabinet. If the value of hydrogen sulfide decreases to less than $10 \mathrm{ppm}$, the device stops the operation of the fan and ion clusters and unlocks the reagent cabinet door.

Figure 5 shows the operation of the device according to nitrogen dioxide. Nitrogen dioxide determines the operation of the device based on $0.03 \mathrm{ppm}$. If the threshold is exceeded, the device operates the fan and ion clusters to cope with dangerous situations. Also, the device stops the devices that were operated if it decreased to less than $0.03 \mathrm{ppm}$.

Figure 6 shows the operation of the equipment according to the sulfur dioxide gas. If the sulfur dioxide gas is more than $0.02 \mathrm{ppm}$, it identifies the dangerous situation and operates the devices. Also, if the sulfur dioxide gas is reduced to less than $0.02 \mathrm{ppm}$, it identifies it as a safe situation and stops the devices

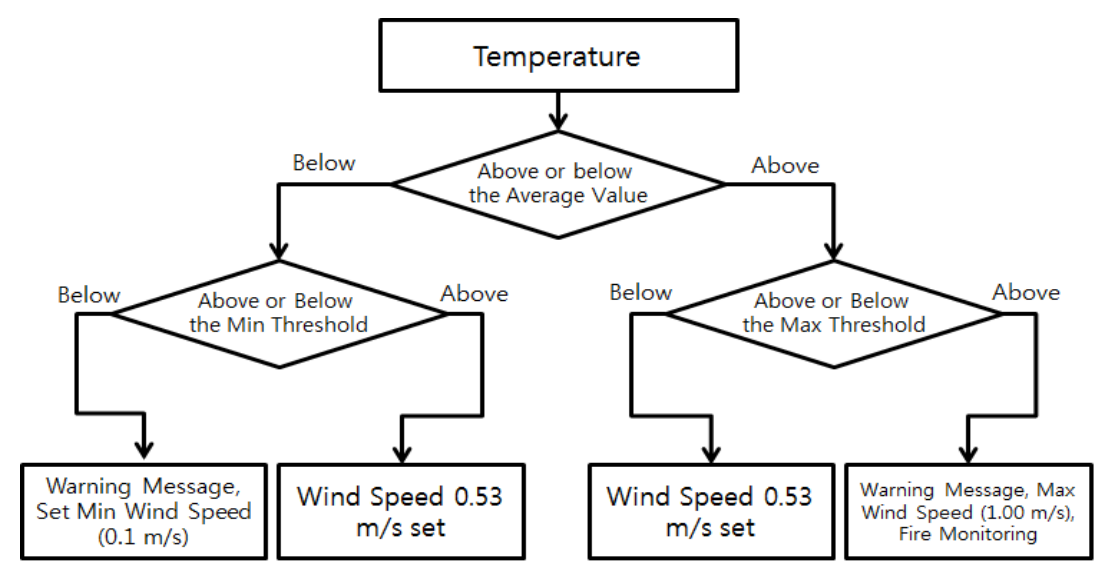

Figure 2. Device operation according to temperature

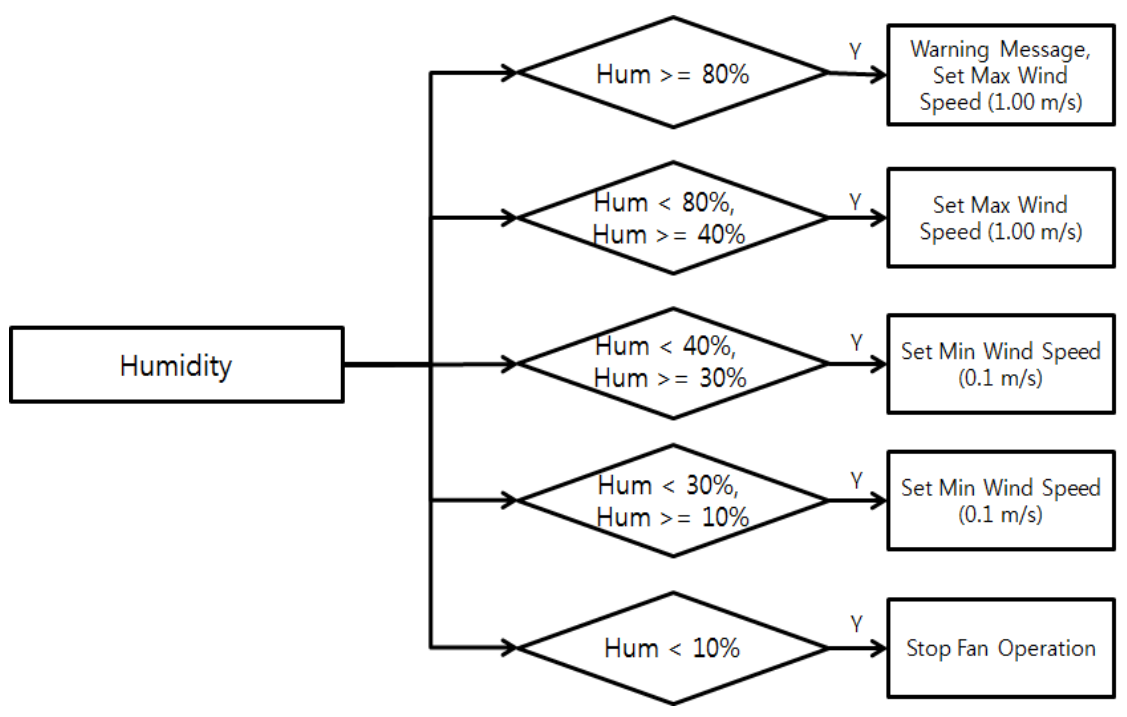

Figure 3. Device operation according to humidity 


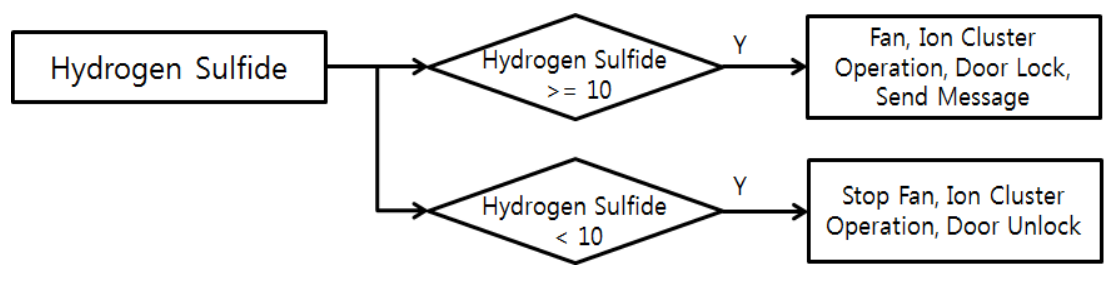

Figure 4. Device operation according to hydrogen sulfide

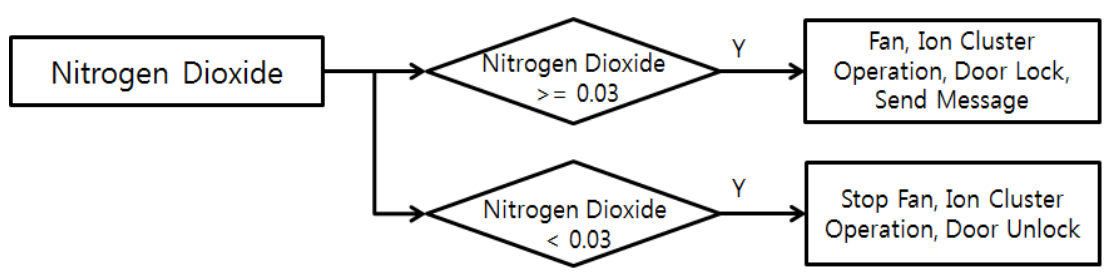

Figure 5. Device operation according to nitrogen dioxide

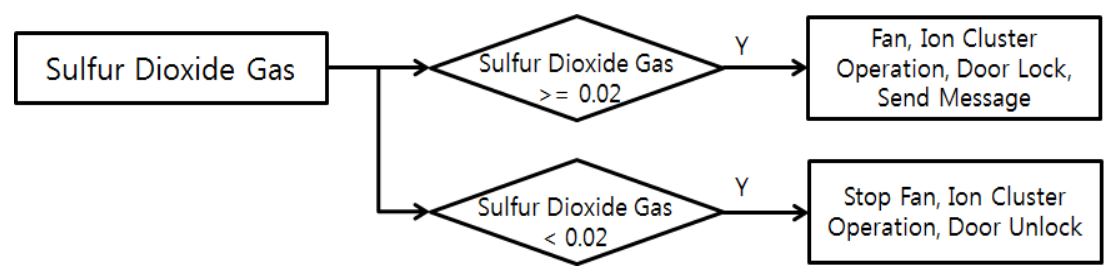

Figure 6. Device operation according to sulfur dioxide gas

Table 1 shows a transmission frame in which collected sensor data are integrated into one and transmitted to the master node. The temperature value was set to a value of 5 bytes because it can be measured up to a maximum of three digits and includes up to the first decimal place. Also, the humidity is set to 3 bytes because it is measured up to $100 \%$. In the gas, hydrogen sulfide, nitrogen dioxide, and sulfurous acid gas each have a value of 4 bytes. Magnetic, fire detection, and smoke detection sensors are set to 1 byte because they identify ' 0 ' or ' 1 ' value. Table 2 shows the device status data transmission frame. We used fan, ion clusters and lamps, respectively. Fan is set to 4byte because it shows up to the second decimal place. Ion cluster and electric lamp are set to 1 byte to identify whether they operate through ' 0 ' or ' 1 ' value.

Table 1. Sensor data transmission frame

\begin{tabular}{ccccccc}
\hline Sensor & Temp & Hum & Gas & Magnetic & Flame & Smoke \\
\hline Byte & 5byte & 3byte & 12byte & 1byte & 1byte & 1byte \\
\hline
\end{tabular}

Table 2. Device status transmission frame

\begin{tabular}{cccc}
\hline Device & Fan & Ion Cluster & Lamp \\
\hline Byte & 4byte & 1byte & 1byte \\
\hline
\end{tabular}

Figure 7 shows a flow chart of the system. The internal environment data of the reagent cabinet measured through the sensors are integrated into one data transfer frame and then transferred to the master node. The master node verifies whether a dangerous situation occurs through transmitted sensor data. The master node identifies dangerous situations by temperature, humidity, and gas if a dangerous situation occurs and automatically operates devices that can respond to these situations. It also continuously sends sensor data and device status to the server and updates it in the database. After a dangerous situation occurs and the device's operation command is transmitted, the device continuously checks whether the dangerous situation has been terminated. If the dangerous situation is terminated, it stops the operation of the devices. 


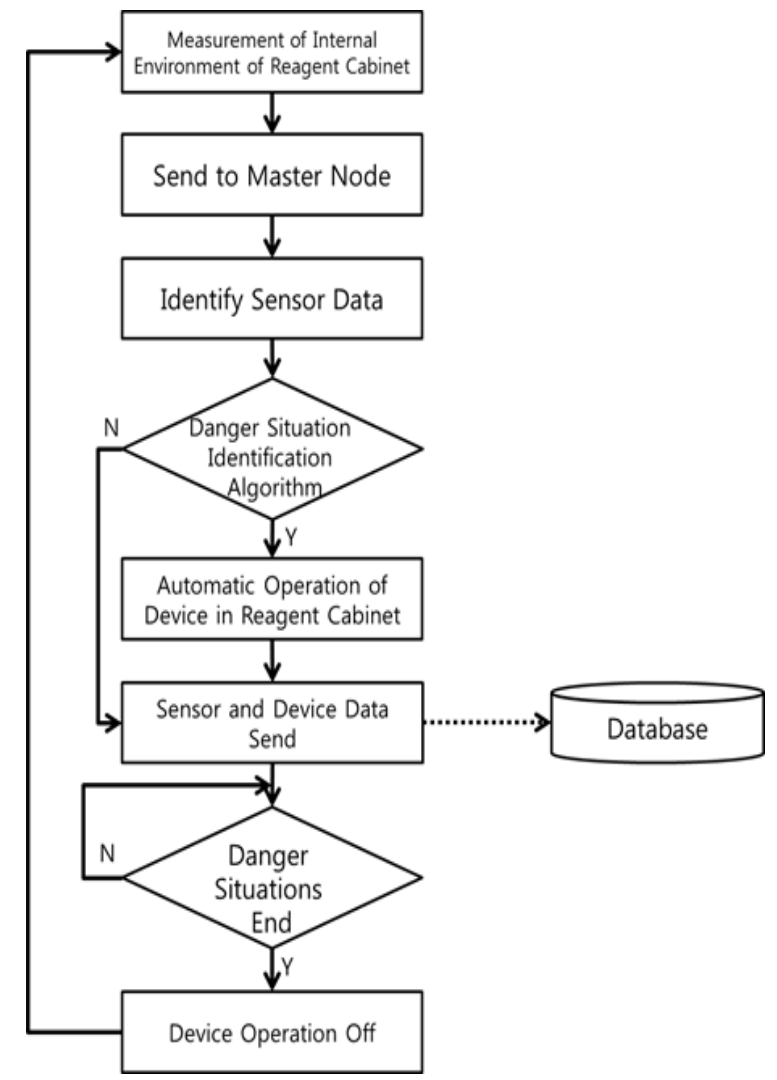

Figure 7. System flow chart

\section{SYSTEM IMPLEMENTATION}

This chapter deals with the implementation of a system that identifies dangerous situations in reagent cabinets and automatically operates the device. Table 3 shows the system implementation environment. In the proposed system, a control board based on Windows CE 6.0 was used to check the real time status of the reagent cabinet. Galaxy S6 is used for remote monitoring and control in applications. On the PC, I built the database through APM Setup. To measure the internal environment of the reagent cabinet, the sensors used temperature, humidity, multi-channel gas, fire detection, smoke detection, and magnetic sensors.

Figure 8 shows the main page of the system implemented through the Windows CE board. You can monitor the real-time temperature and humidity of the reagent cabinet, the values for the gas sensors, and check the status of the fan, ion clusters and doors. The status of the device indicates that the device is in operation when the Toggle Button is enabled and the device is inoperable if it is disabled

Figure 9(a) shows the main page of the Android application. The main page consists of temperature, humidity, gas, and instrument status. Temperature and humidity indicate the temperature and humidity measured in the inside of the reagent in real time, and the gas monitors hydrogen sulfide, nitrogen dioxide, and sulfur dioxide. The device indicates the current operating state. Also, it consists of control page for remote control of the reagent cabinet, and a view page where you can view information about the dangerous situation in the reagent cabinet.

Figure 9(b) shows the view page of the Android application. The view page allows you to monitor messages sent when a dangerous situation occurs in the reagent cabinet. A message about a dangerous situation indicates information about the year, month, day, hour, and dangerous situation that occurred. Messages are also automatically deleted after three months of storage.

Figure 9(c) shows the control page of the Android application. The manager can set the temperature and humidity and remotely control the status of the device. You can also set the desired wind speed when you turn on the fan. After the setting is completed, you click the Set button to control the reagent cabinet according to the values set by the manager. 
Table 3. Development environments from machine

\begin{tabular}{cc}
\hline Type & Composition \\
\hline Tools & Edit Plus, Android Studio, APM Setup7, Windows CE 6.0, Arduino, FCM \\
PC & Window 7 Enterprise K 64bit, Intel i5-4460, 8GB RAM \\
Sensor & Temperature, Humidity, Multichannel Gas, Flame, Smoke, Magnetic \\
Device & Arduino UNO, Window CE, Galaxy S6 \\
\hline
\end{tabular}

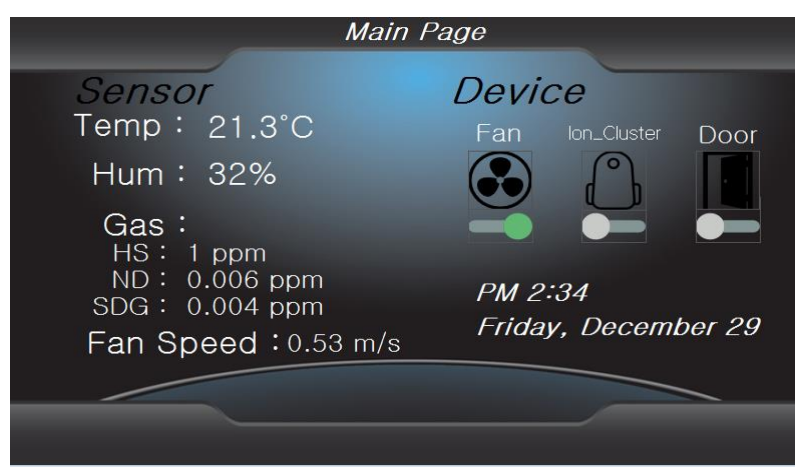

Figure 8. Application main page

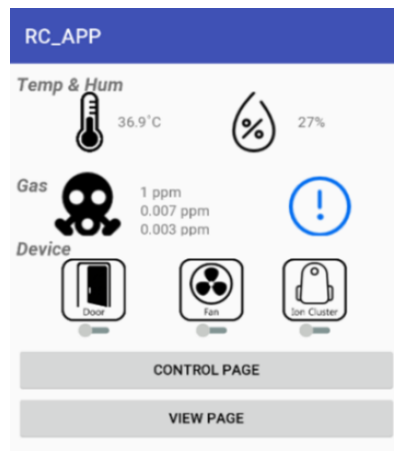

(a)

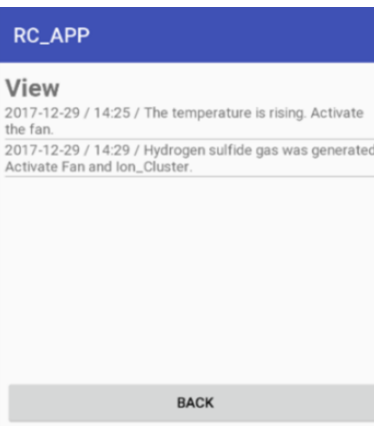

(b)

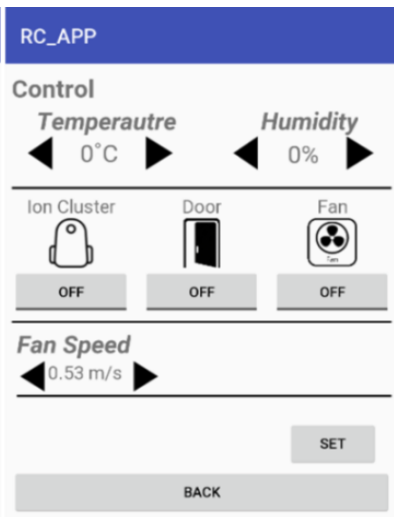

(c)

Figure 9. Android application, (a) Main page, (b) View page, (c) Control page

\section{REVIEW}

This section deals with the comparison between the existing system and the proposed system. The data set for the experiment is used in the existing system and the proposed system in the same way. The experiment confirms how much time it takes for the device to operate when the value of hydrogen sulfide is out of the threshold. The number of experiments is 20 each. Table 4 shows the existing system and the data set of the proposed system.

Table 4. System experiment dataset

\begin{tabular}{cc}
\hline Dataset & Description \\
\hline $\begin{array}{c}\text { Hydrogen Sulfide } \\
\text { Count }\end{array}$ & 1 to 20 ppm \\
\hline
\end{tabular}

As a result of the experiment in Figure 10, when the existing system was applied, the dangerous situation occurred and the time required for the device to operate was about 4 seconds on average. These results may be more time consuming due to the situation of the manager, because it can be different 
depending on how quickly the manager responds to the dangerous situation. The proposed system took an average of about 2.2 seconds until a dangerous situation occurred and the device became active. Unlike the existing system, the system automatically selects and operates the device in the system without manual control by the manager. As a result, it is confirmed that it is possible to cope more quickly than the manual control by the manager like the existing system.

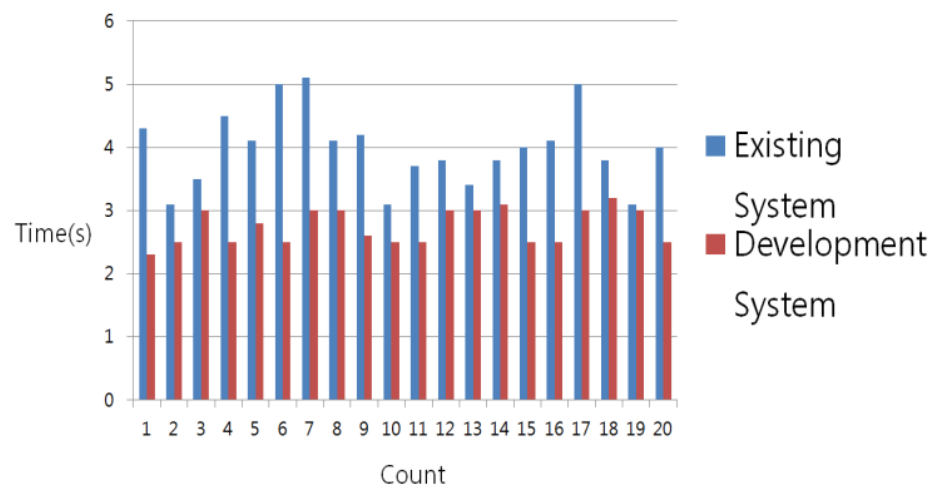

Figure 10. Experimental results of system without danger priority applied

\section{CONCLUSION}

The existing system transmits the internal environment data of the reagent cabinet to the server, identifies the dangerous situation, and sends a notification message to the manager. The manager can identify the dangerous situation through the notification message and operate the device through the control board or application to cope with this situation[17-20]. As described above, when the manager manually controls the devices, there is a problem that it is difficult to quickly cope with a dangerous situation when the manager is not present. Also, since the server performs all the operations that occur in a plurality of reagent cabinets, the server operation speed may become slower as the number of reagent cabinets increases[21-25].

In order to solve these problems, this paper proposes a system that identifies dangerous situations in a reagent cabinet and automatically operates the device. In the reagent cabinet, sensor data and device status are collected through the sensor node and transmitted to the master node. The master node identifies the dangerous situation through the transmitted sensor data and then operates the device automatically. In addition, by continuously transmitting sensor data and device status to the server, the real-time status of the reagent cabinet can be confirmed from the application or control board. Applications can monitor and control the reagent cabinet status in real time through remote monitoring and control. Future research will be able to reduce the time consumed in identification of dangerous situation or device operation command by implementing reagent cabinet module that integrates control board, sensor node and master node into one.

\section{ACKNOWLEDGEMENTS}

This research was supported by The Leading Human Resource Training Program of Regional Neo industry through the National Research Foundation of Korea(NRF) funded by the Ministry of Science, ICT and future Planning(No. 2016H1D5A1911091). This work was supported by the research grant of Paichai University in 2019.

\section{REFERENCES}

[1] J. O. Westgard, "Useful measures and models for analytical quality management in medical laboratories," Clinical Chemistry and Laboratory Medicine (CCLM), vol. 54, no. 2, pp. 223-233, 2016.

[2] H. I. Jung and K. Y. Chung, "Knowledge-based dietary nutrition recommendation for obese management," Information Technology and Management, vol. 17, no. 1, pp. 29-42, 2016.

[3] C. I. Cucoranu, "Laboratory Information Systems Management and Operations," Clinics in laboratory medicine, vol. 36 , no. 1, , pp. 51-56, 2016.

[4] R. Roos, K. Brown and T. Torabi, "LUS-a tablet-based NFC system to facilitate instant database archival of laboratory assessment", Australasian Journal of Engineering Education, vol. 21, no. 2, pp. 74-80, 2016. 
[5] A. Merabet, K. T. Ahmed, H. Ibrahim, R. Beguenane and A. M. Ghias, "Energy management and control system for laboratory scale microgrid based wind-PV-battery," IEEE Transactions on Sustainable Energy, vol. 8, no. 1, pp. 145-154, 2017.

[6] H. W. Kim, E. G. Lee and D. W. Kwon, "Chemical laboratory safety management service using IoT sensors and open APIs," in Information and Communications (ICIC), 2017 International Conference on, 2017.

[7] K. Cao, H. Lee and J. Jung, "Task Management System According to Changes in the Situation Based on IoT," Journal of Information Processing System, vol.13 no.6, pp. 1459-1466, 2017.

[8] K. Cao, I. Kang, H. Choi and H. Jung, "Reagent Cabinet Management System Using Danger Priority", Journal of Information and Communication Convergence Engineering, vol. 15, no. 4, pp. 227-231, 2017.

[9] Y. Evchina, J. Puttonen, A. Dvocyanchikova and J. L. M. Lastra, "Context-aware knowledge-based middleware for selective information delivery in data-intensive monitoring system," Engineering Applications of Artificial Intelligence, vol. 43, pp. 111-126, 2015.

[10] N. J. Cho and Y. G. Ji, "Indoor IoT Monitoring System based on Visible Light Communication using Smart Phone," Journal of the Korean Institute of Telematics and Electronics, vol. 54, no. 4, pp. 35-43, 2017.

[11] H. J. Lee, H. W. Choi and H. K. Jung, "Reagent Cabinet Hazard Situation Identification System Utilizing Multiple Sensor Data," Journal of the Korea Institute of Information and Communication Engineering, vol. 22, no. 1, pp. 63-68, 2018.

[12] S. J. Lee and J. G. Hong, "Measuring the Greenhouse Gas Emission Reduction and Management System Using Bluetooth Sensor Node," Journal of Information and Communication Convergence Engineering, vol. 17, no. 5, pp. 1095-1100, 2014.

[13] Y. J An, D. H. Kim, J. H. Lee and B. J. Lee, "Indoor Environment Control System Utilizing the Internet of Things," Journal of the KIECS, vol. 12, no. 4, pp. 645-650, 2017.

[14] W. Y. Lee, H. M. Ko, J. H. Yu and B. Sim, "An Implementation of Smart Dormitory System based on Internet of Things," Journal of Korean Institute of Intelligent Systems", vol. 26, no. 4, pp. 295-300, 2016.

[15] M. J. Song, "A Study on Business Types of IoT-based Smarthome: Based on the Theory of Platform Typology," The Journal of the Institute of Internet, Broadcasting and Communication, vol. 16, no. 2, pp. 27-40, 2016.

[16] N. J. Cho and Y, G. Ji, "Analysis of Safety Management Condition \& Accident Type in Domestic and Foreign Laboratoy," Journal of the Engonomics Society of Korea, vol. 35, no. 2, pp. 97-109, 2016.

[17] X. Lie, J. Li and X. Li, "Study of Dynamic Risk Management System for Flammable and Exposive Dangeous Chemicals Storage Area," Journal of Loss Prevention in the Process Industries, vol. 49, pp. 983-988, 2017.

[18] Y. H. Cho, "Smart Windows and Doors Platform for Providing Optimized Inner Environment", Journal of Korean Intitute of Intelligent Systems, vol. 25, no. 5, pp. 464-469, 2015.

[19] J. H. Kim, "Development of U-Building Fire Safety Management System," Fire Science and Engineering, vol. 30, no. 1, pp. 74-80, 2016.

[20] C. S. Oh, M. S. Seo, J. H. Lee, S. H. Kim, Y. D. Kim and H. J. Park, "Indoor Air Quality Monitoring System in the IoT Environment," The Journal of Korean Institute of Communication and Information Science, vol. 40, no. 5, pp. 886-891, 2015.

[21] J. W. Park, D. S. Kim and N. K. Joo, "Indoor Environment Monitoring and Controlling System Design and Implementation based on Interent of Things," Journal of the Korea Institute of Information and Communication Engineering, vol. 20, no. 2, pp. 367-374, 2017.

[22] Y. S. Moon, J. W. Jung, S. P. Choi, T. H. Kim, B. H. Lee, J. J. Kim and H. L. Choi, "Real-Time Management System of Reefer Container based on IoT," Journal of the Korea Institute of Information and Communication Engineering, vol 19, nio. 9, pp. 2093-2099, 2015.

[23] C. C. Shih, R. S. hong and S. K. Lee, "Investigation of Lab Fire Prevention Management System of Combining Root Cause Analysis and Analytic Hierarchy Process with Event Tree Analysis," Machematical Problems in Engineering 2016.

[24] S. Bruckner, J. L. Marendaz and T. Meyer, "Using Very Toxic or Especially Hazardous Chemical Substances in a Research and Teaching Institution," Safety Science, vol. 88, pp. 1-15, 2016.

[25] J. Back, "Secure Pre-authentication Schemes for Fast Handoff in Proxy Mobile IPv6," Journal of Information and Communication Convergence Engeering, vol. 14, no. 2, pp. 89-96, 2016.

\section{BIOGRAPHIES OF AUTHORS}

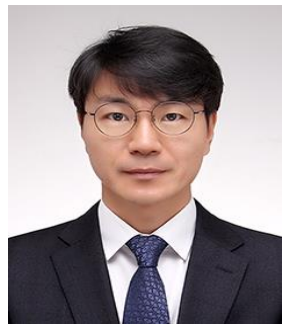

Changsu Kim, he received his B.S., M.S., and Ph.D. degrees from the Department of Computer Engineering of Paichai University, Korea, in 1996, 1998, and 2002, respectively. From 2005 to 2012, he worked for the Department of Internet at Chungwoon University as a professor. Since 2013, he has worked in the Department of Computer Engineering at Paichai University, where he now works as a professor. His current research interests include multimedia document architecture modeling, web 2.0 , and the semantic web. 

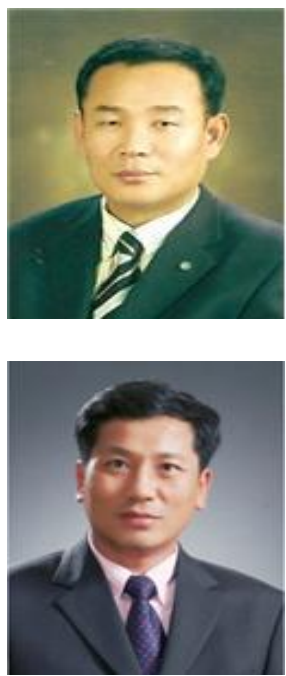

Hongyoul Kim, he received the B.S. degree in 2015 from the Department of Social Welfare of Honam University and M.S. degree in 2019 from the Department of Computer Engineering of Paichai University, He is currently a Doctorate course in Department of Computer Engineering of Paichai University. Korea. Since 1999, he has worked at Kwang jin Co.,Ltd. where he now works as a representative Director. His current research interests include multimedia document architecture modeling, IoT and AI.

Hoekyung Jung, he received the M.S. degree in 1987 and Ph. D. degree in 1993 from the Department of Computer Engineering of Kwangwoon University, Korea. From 1994 to 1995, he worked for ETRI as a researcher. Since 1994, he has worked in the Department of Computer Engineering at Paichai University, where he now works as a professor. His current research interests include multimedia document architecture modeling, information processing, information retrieval, machine learning, bigdata, and IoT. 